\title{
A REFLECTION SPECTROPHOTOMETRIC STUDY OF ULTRA- VIOLET ERYTHEMA AND PIGMENTATION
}

\author{
By M. T. JANSEN \\ (From the Department of Histology and Microscopic Anatomy, State University, Utrecht, \\ The Netherlands)
}

(Submitted for publication November 25, 1952; accepted June 24, 1953)

Skin reactions to ultraviolet radiation have been studied several times by making histological preparations at different intervals $(1,2,3)$ but this method, though indispensable, needs amplification by less destructive procedures, e.g., by spectrophotometry.

\section{METHODS AND MATERIALS}

It has been established that the normal skin reflection measured on consecutive days shows considerable fluctuation, mainly because of differences in the width of the blood vessels and in the degree of oxygenation of the blood. This fluctuation precludes the prolonged observation of slight degrees of erythema or of other alterations in the color of the skin, if reference is made to a standard as is done in most spectrophotometric procedures.

In order to overcome this difficulty we have chosen to compare the experimental skin area with closely adjacent normal areas of the skin by recording successively the reflection of normal and altered areas: An optical system projects an image of the exit slit of a monochromator onto the skin, i.e., it illuminates a small rectangular area of the skin with monochromatic light of any desired wave length. The slit image, which measures $3 \mathrm{~mm}$. in height and $0.2 \mathrm{~mm}$. in breadth, is made to scan at a speed of $2 \mathrm{~mm}$. per second, a skin area of 3 by $5 \mathrm{~mm}$. (see Figure 1). The light scattered back by the skin is collected in an integrating sphere and measured by means of a vacuum photocell. The amplified photocurrent is recorded on a photographic paper strip moving synchronously with the illuminating spot on the skin. Moreover, after each sweep, zero reflection and the reflection of an invariable standard is recorded automatically.

Figure 2 gives two typical registrations obtained with different parts of the skin. In these recordings the zero lines $(0)$ connect the zero reflection registrations whereas the lines St are on the level of the standard reflection at the wavelength used. In the left-hand recording the skin reflection line, though sloping, is straight: no local alteration in skin reflection has been recorded by the scanning slit image. In the right-hand registration, however, the depression in the skin line clearly marks the presence of an area of reduced reflection. To what extent these recordings can be used in the analysis of ultraviolet irradiation effects will be discussed in the next section.

The investigation, for which the method described in the foregoing section was developed, aimed at the solution of the following problem: are erythemata (and pigmentations) yielded by ultraviolet irradiation of $300 \mathrm{~m} \mu$ exactly like those caused by $257 \mathrm{~m} \mu$ ? It has been generally assumed that there is only one erythematogen mechanism in the ultraviolet range below $300 \mathrm{~m} \mu$, the "dip" in the action spectrum at $280 \mathrm{~m} \mu$ being caused by the absorption of the horny layer. Recently, Blum and Terus (4) have suggested a modification of the $300 \mathrm{~m} \mu$ erythema by a direct action of the rays on the capillaries themselves, whereas Rottier and Mullink $(5,6)$ advocate the existence of two entirely different photochemical mechanisms elicited by 300 and $257 \mathrm{~m} \mu$, respectively.

Precise comparison of the two kinds of erythema (and pigmentation) should, in our opinion, be of great value in this controversy as well as adding to our knowledge of skin physiology in general.

For this purpose small areas ( 1 by $5 \mathrm{~mm}$.) of moderate erythema were generated by irradiation with 257 and $302 \mathrm{~m} \mu$, respectively (mercury arc, quartz spectrograph). All irradiations were performed on the forearm of one dark blonde male subject. The reflection of the irradiated areas and of the adjoining normal skin was recorded at regular intervals after the irradiation.

As regards the choice of the wave lengths at which to perform the measurements, we were guided by the known peculiarities of the transmission spectra of the pigments to be expected, i.e., primarily hemoglobin (7) and melanin $(8,9)$ (Figure 3 ).

Though it is obviously important to choose the wave

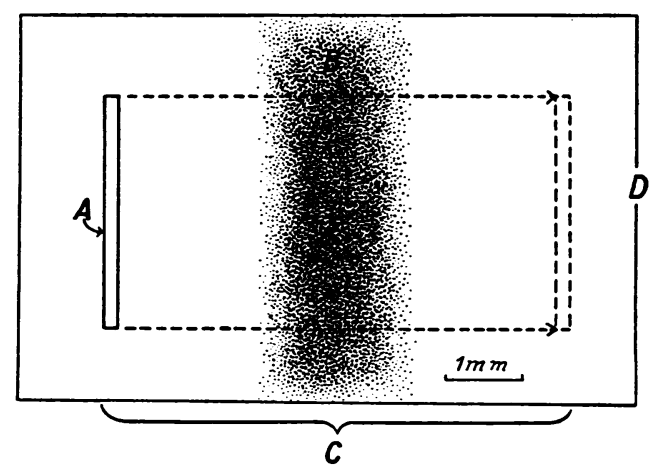

Fig. 1. Diagram of Investigated Skin Area

$A$ : scanning spot of light projected onto the skin surface; B : colored skin area; C: width of skin area scanned by $A ; D$ : border of the window in the integrating sphere to which the skin is applied. 

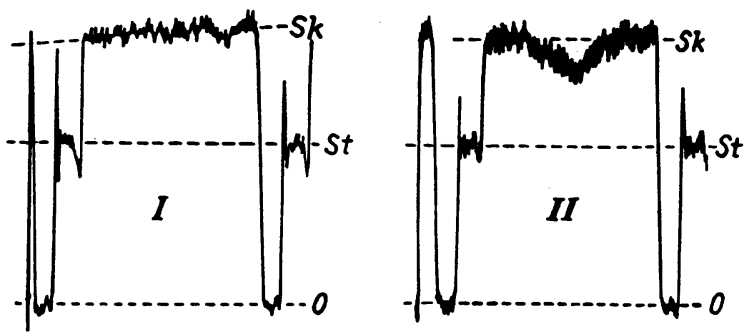

Fig. 2. Typical Curves Obtained with the Method

Right : erythema or pigmentation present; left: no local coloring of the skin; $0:$ 'zero line; St: level of the reflection of the standard; Sk: normal skin reflection level (skin line).

lengths to be measured at strategical points (designated by dots) along the hemoglobin curve, the same cannot be said in the case of melanin. Note that the transmission of a melanin solution at $600 \mathrm{~m} \mu$ is only slightly different from that at $579 \mathrm{~m} \mu$, whereas in the case of hemoglobin there is a great difference between the transmission values at these two wave lengths.

The two rows of curves of Figure 4 are actual registrations of the reflection of two differently colored areas of the skin, the top row referring to an erythematous area, the bottom row to a preponderantly pigmented streak. Both areas are represented by a depression in the $520 \mathrm{~m} \mu$ curves but in the 600 and $313 \mathrm{~m} \mu$ curves only the pigmented area is recognizable. That the erythema is not visible in the $600 \mathrm{~m} \mu$ curve can be readily understood as blood pigment absorbs only a small fraction of the light of this wave length. The $313 \mathrm{~m} \mu$ graph fails to record the presence of an increased amount of blood in the capillaries, as the upper layer of the epidermis forms an effective screen for this wave length. All or most of the little light of this wave length received back from the skin was reflected by the surface and scattered back by the uppermost layers and did not traverse the capillaries of the dermis. As the melanin, responsible for the depressions in the bottom row of curves, was located in the upper layers of the epidermis, its presence was also shown by the $313 \mathrm{~m} \mu$ recording. Note the different shapes of the depressions at 600,520 , and $313 \mathrm{~m} \mu$, which might suggest different distributions of the light absorbing substances. Yet, this difference is more plausibly explained by the fact that in the epidermis the penetrating power of light diminishes with decreasing wave lengths. In the case of $600 \mathrm{~m} \mu$ the bulk of the light thrown back by the skin comes from the scattering collagenous fibers of the dermis, whereas we have seen that light of $313 \mathrm{~m} \mu$ is preponderantly reflected by the uppermost layers. As in the case of the curves of Figure 4 (II), the screen of pigment was located in the upper layers of the epidermis; the $313 \mathrm{~m} \mu$ light, being scattered back from the same plane, can be considered to trace the distribution of the pigment most truly, whereas the depression in the $600 \mathrm{~m} \mu$ recording will necessarily give a distorted picture of the distribution of pigment in the area. These considerations have been borne out by experiments with neutral grey screens of accurately known geometry applied to the surface of the skin.

\section{RESULTS}

With these observations in mind we now turn to the results of our measurements.

\section{Reflection spectra of ultraviolet erythema and pigmentation}

The essential feature of our method lies in the comparison of the altered skin with normal skin.

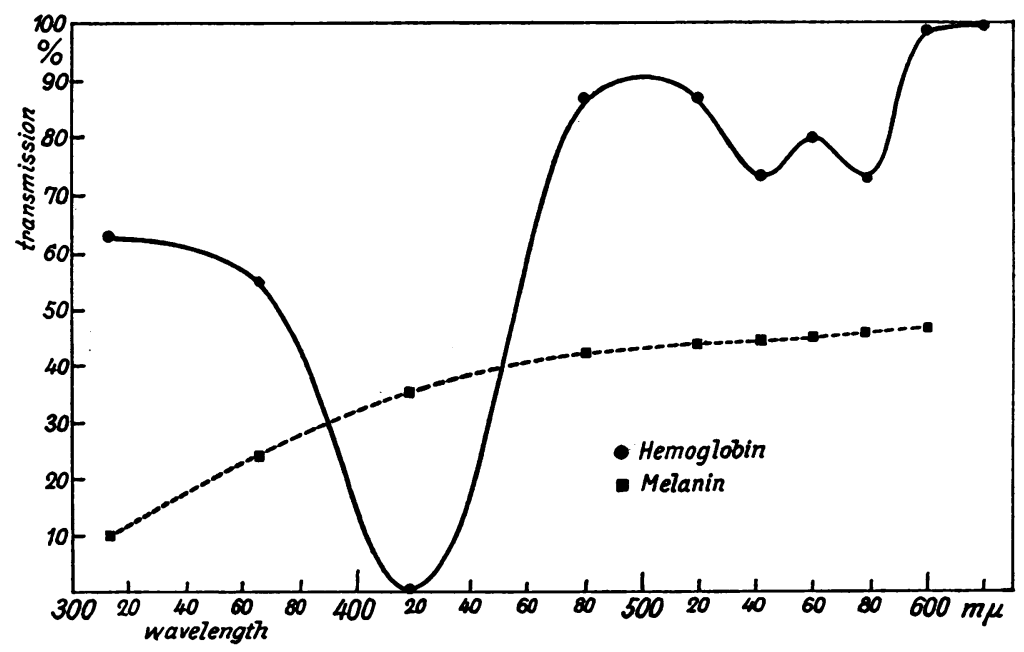

Fig. 3. Transmission Spectra of 75.6 Per Cent Oxygenated Hemoglobin in a Concentration and Layer Thickness Comparable to a Blood Fily of $10 \mu$ Thickness (Drabkin and Schmidt (7)) and of Mraninin "in Alkaline Solution" (Edwards, Finkelstein, and Duntley (9)) 
Accordingly, the measured values of reflection in the erythematous and pigmented areas have been plotted as percentages of "normal skin reflection" (i.e., the mean of the reflection values of the "normal" skin areas on both sides of the area under investigation ${ }^{1}$ ).

In Figure 5, the reflection of the center of an irradiated area $\left(257 \mathrm{~m}^{\mu}\right)$, at different intervals after the irradiation, has been plotted in this manner. The one day curve apparently shows the presence of an excess of blood pigment in the irradiated area: erythema. The curve resembles the transmission curve of Figure 3, but it cannot be considered as such because of the scattering and ab-

1 By comparing the observed normal skin values with the standard reflection values we have found a normal reflection spectrum not essentially different from those observed by other workers in the field. sorption of light in the superficial layers of the skin. These effects cause a distortion of the transmission curve which is greatest at the short wave length end of the spectrum.

On the first day after irradiation melanin is not increased as reflection at 600 and $620 \mathrm{~m} \mu$ has remained normal. After four days, however, the influence of increased melanin pigment in the irradiated area on the reflection spectrum is indicated by the lowering of the 600 and $620 \mathrm{~m} \mu$ values and of the whole curve especially towards the shorter wave lengths. After eight days the greatest difference from the foregoing curve is found in the short wave length region.

A further increase in pigmentation cannot be responsible for this, as the values at 600 and $620 \mathrm{~m} \mu$ have remained unaltered. Without reverting to the assumption of an alteration of the absorption

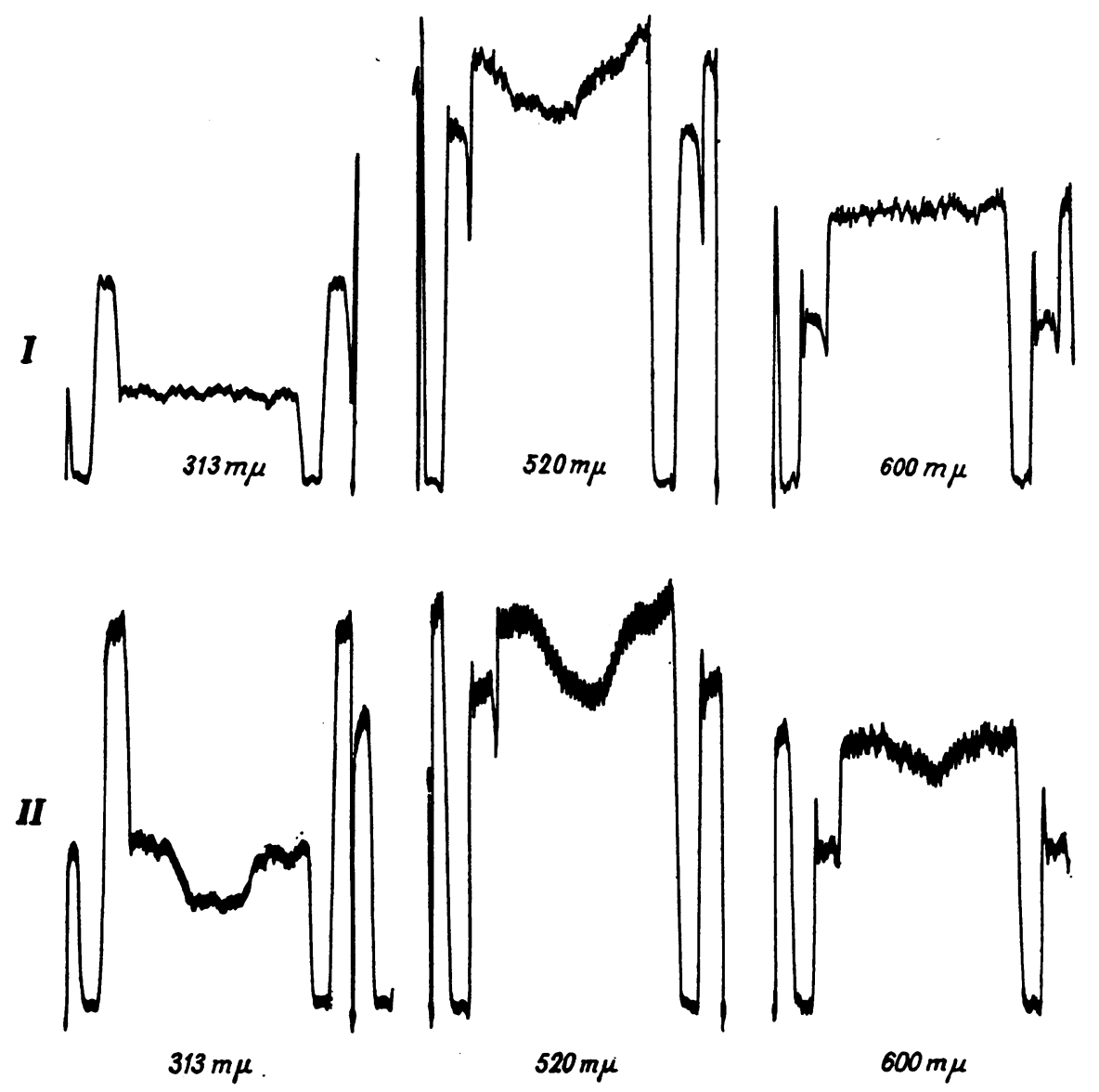

Fig. 4. Selected Typical Curves Illustrating the Difference between the Spectral Reflections of Erythematous (Top Row) and Pigmented Areas (Botrom Row) The numbers refer to the wave lengths at which the curves were recorded. 


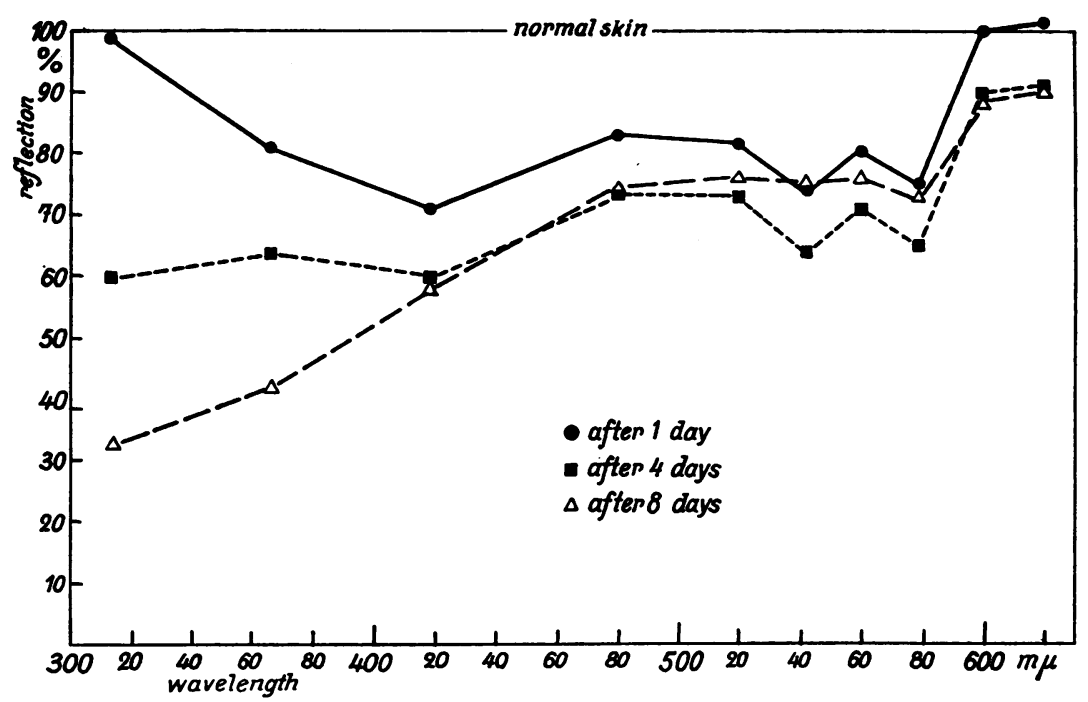

Fig. 5. Spectral Reflection of the Center of an Area of the Skin Irradiated with $257 \mathrm{~m} \mu$ Ultraviolet Light, Expressed in Percentages of the Normal Skin Reflection

See text for further explanation.

spectrum of the pigment, the difference between the fourth and eighth day curves can be interpreted as follows: the melanin formed in the first days after irradiation is located in the deeper layers of the epidermis. Thus, the low transmission of melanin at short wave lengths does not greatly influence the skin reflection curve as has been discussed in the case of hemoglobin in the capillaries. Pigment, however, migrates to the surface of the epidermis in the course of a few days. Accordingly it gradually gains influence on the short wave length reflection values of the area.

Note that after eight days the absorption of hemoglobin is still recognizable though the $418 \mathrm{~m} \mu$

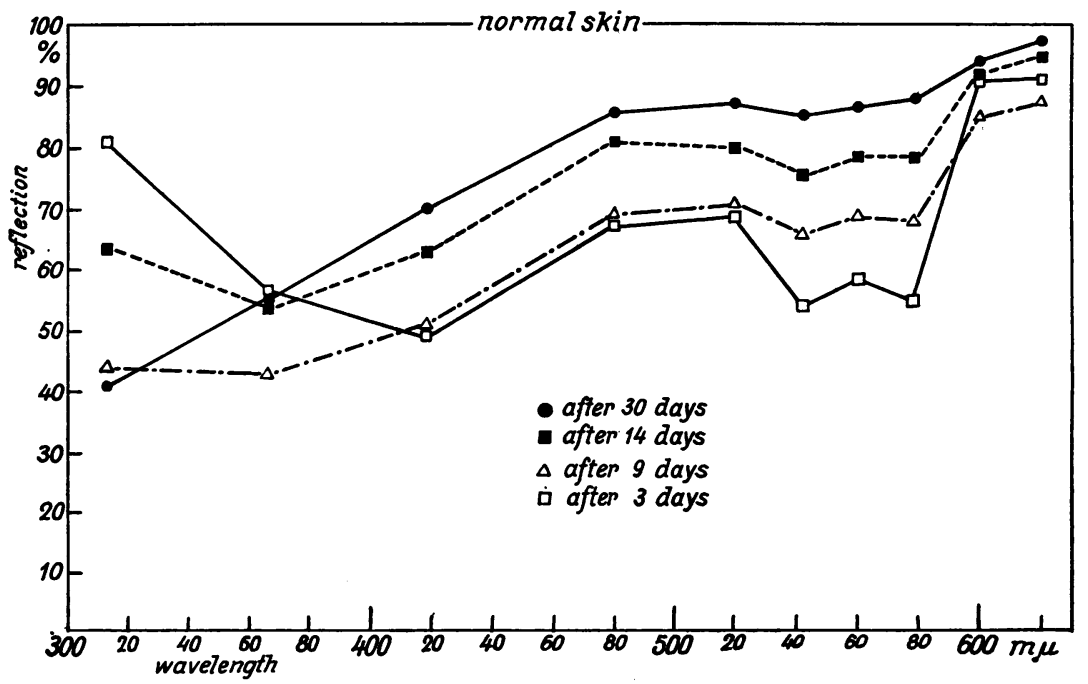

Fig. 6. Spectral Reflection of the Center of an Area of the Skin Irradiated with $300 \mathrm{~m} \mu$ Ultraviolet Light, Expressed in Percentages of the Normal Skin Reflection

Note persistence of erythema characteristics after as many as 30 days. See text for further explanation. 


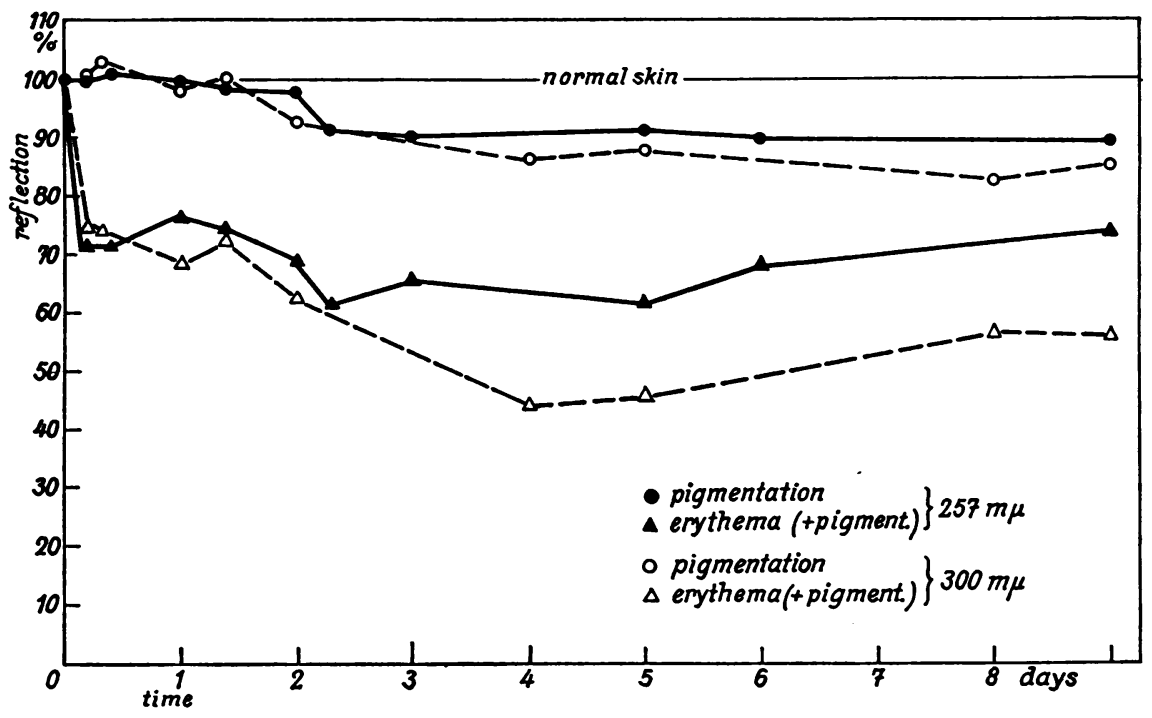

Fig. 7. Skin Reflection at 579 (Erythema + Pigmentation) and $600 \mathrm{~m} \mu$ (Pigmentation Only) in Center of Areas Irradiated with 257 and $300 \mathrm{~m} \mu$, RESPECTIVELY

Reflection is in percentages of normal skin reflection, time in days.

depression has been obscured by the melanin absorption.

Comparison of these curves with similar recordings of areas irradiated with $300 \mathrm{~m} \mu$ instead of $257 \mathrm{~m} \mu$ yields no significant differences.

Figure 6 shows the reflection spectra of a 300 $\mathrm{m} \mu$ ultraviolet erythema which has been followed over a period of 30 days. The ninth and third day curves differ in the same way as do the eighth and fourth days curves of Figure 5. The differences between the curves obtained at the ninth and fourteenth days are particularly interesting, as scaling of the irradiated area took place on the eleventh and twelfth days. The curves suggest that scaling involves a loss of superficially situated pigment. The erythemal intensity, as judged by the difference of the reflection values at 600 and 579 $\mathrm{m} \mu$, seems not to have been greatly influenced by the scaling.

The erythema is still discernible in the curve taken as many as 30 days after the irradiation. The same has been found to be the case after 257 $\mathrm{m} \mu$ irradiations.

\section{Mode of appearance of erythema and pigmenta- tion after irradiation}

In Figures 7 and 8, erythemal and pigmentation values have been plotted against time. For these curves, the $579 \mathrm{~m} \mu$ reflection values have been used to denote the degree of erythema, although, after the first few days, melanin pigment, too, will influence the reflection values at this wave length. The amount of melanin could be assessed separately with the aid of the reflection values at 600 or 620 $\mathrm{m} \mu$.

If one follows the course of erythema and pigmentation at daily intervals after the irradiation (see Figure 7), no differences between the results of 257 and $300 \mathrm{~m} \mu$ irradiation are noted, provided that ultimate degrees of erythema and pigmentation are moderate and not too widely divergent.

Measurements repeated at hourly intervals, however, consistently showed the $257 \mathrm{~m} \mu$ erythema to appear several hours earlier than the $300 \mathrm{~m} \mu$ redness. Figure 8 gives a typical example. The

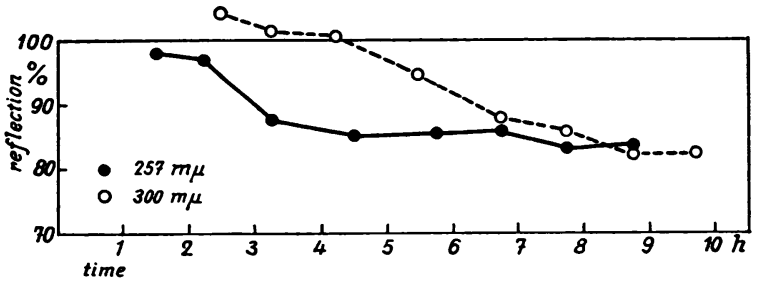

Fig. 8. Degree of Erythema (as Measured by the $579 \mathrm{~m} \mu$ Reflection in Percentages of Normal Skin REFLECTION) AT HOURLY INTERVALS AFTER IRRADIATION WITH 257 OR $300 \mathrm{~m} \mu$ ULtraviolet LIGHT 
temporary increase in reflection of the irradiated area will be discussed below.

\section{Distribution of the pigment in the irradiated skin areas}

The information our method yields on the distribution of pigments in the area scanned should be used with extreme caution. Even a sharply delineated absorbing screen, with its borders exactly parallel to the rectangular scanning spot, will not yield a perfectly rectangular depression in the reflection curve. First, the scanning spot of light is not infinitely narrow; secondly, in the case of the skin the light does not always come back from the plane of the screen. Furthermore, the mean depth of penetration of the backscattered light varies with the wave length; moreover, the position of the two screens considered (viz., the distended capillaries and the augmented pigmentation) may be different, too. The latter difficulty cannot be circumvented but we can cope with the former by comparing recordings taken at one wave length only.

In Figure 9 of three recordings of one and the same area (one, five, and eight days after irradia-

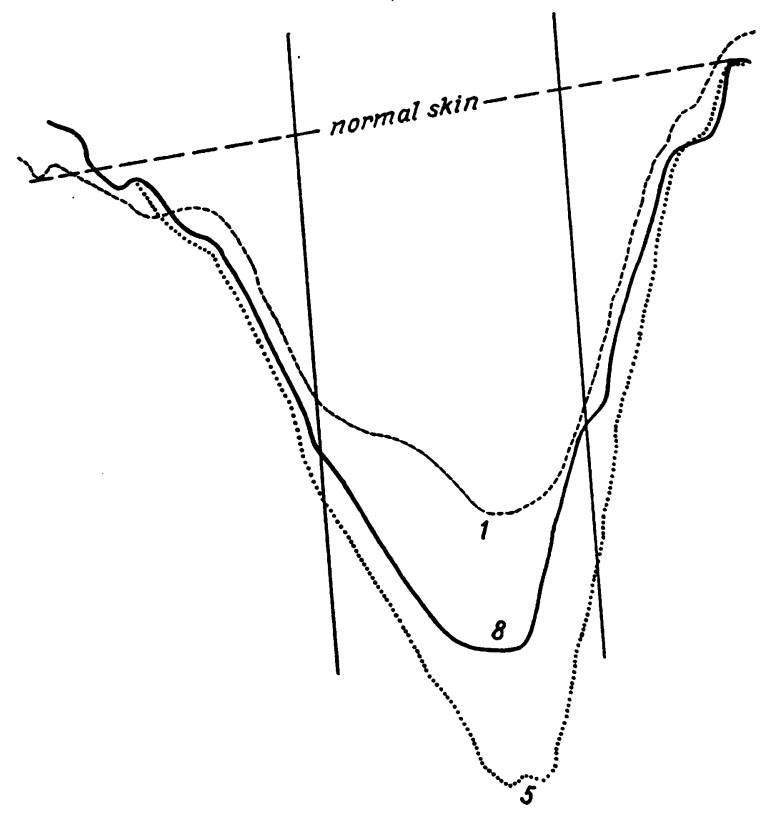

Fig. 9. Superimposed Recordings of aN IrRadiated (300 m $\mu$ ) Skin Area Taken with $520 \mathrm{~m} \mu$ at Intervals of ONe, Five, aNd Eight Days after the Irradiation

Distance between vertical lines indicates breadth of irradiated area $(1.5 \mathrm{~mm}$.). tion with $300 \mathrm{~m} \mu$ ) the depressions in the skin lines have been superimposed. The recordings had been made at $520 \mathrm{~m} \mu$, as the skin reflection at this wave length is affected moderately by both erythema and pigmentation. After one day, erythema will be solely responsible for any depression in the curve, whereas pigmentation will dominate after eight days. Yet, the curves indicate an essentially identical distribution of the colored matter irrespective of the interval between irradiation and registration, i.e., irrespective of whether it be erythema or pigmentation. The same result has been obtained after irradiation with $257 \mathrm{~m} \mu$.

The vertical lines indicate the position and the breadth of the area exposed to the ultraviolet light during irradiation. The curve suggests an extension of the erythemal reaction (and of the pigmentation) beyond the borders of the irradiated area, but we cannot be certain on this point, as we do not know to what extent the scattering of light may blur the contours of the colored area. The shapes of the curves of different identically irradiated areas of the skin differed somewhat. Therefore, we could not compare with exactness the distribution of erythema and pigmentation of areas irradiated at $257 \mathrm{~m} \mu$ with those exposed to $300 \mathrm{~m} \mu$.

\section{DISCUSSION}

1. The described method allows comparison, by spectrophotometric means, of very small skin areas (approximately 1 by $4 \mathrm{~mm}$.) with closely adjacent normal skin areas or with a standard. Further reduction of the dimensions of the spot of light will not enhance the resolving power of the method, as the penetration of the skin by the light and the minute irregularities of the skin surface are limiting factors, which are canceled out by the length of the scanning spot.

2. In interpreting the observed phenomena, we have assumed two pigments to increase in quantity as the result of ultraviolet irradiation. That most of the facts can be explained on this assumption in a semi-quantitative manner does not imply of course that subtler methods would fail to detect other light-absorbing substances involved in the reactions.

The results obtained can be summarized as follows : 
Erythema is the first constant result of appropriate ultraviolet doses; the $257 \mathrm{~m} \mu$ erythema is spectrophotometrically identical with the $300 \mathrm{~m} \mu$ erythema. The latter, however, tends to appear later than the former. In both cases, the erythema is shown to persist much longer than is generally assumed. In fact, for the duration of our experiments (45 days) no visible result of ultraviolet irradiation has been found in which erythema was not detectable. Recently, Schulz (10) obtained similar results.

No differences in the modes of appearance and in the spectrophotometric characteristics of 257 and $300 \mathrm{~m} \mu$ pigmentations have been found. Pigmentation always followed and never preceded erythema. There is a close correlation between the areas of erythema and pigmentation.

We found no evidence for the assumption of Keller (1) and Miescher (2), based on histological findings, that the first stages of visible pigmentation can be accounted for solely by a migration of the pigment already present. On the contrary, our findings indicate an early increase of melanin and subsequent migration of the pigment.

Thus, the question originally posed, i.e., "Are erythemata and pigmentations caused by $300 \mathrm{~m} \mu$ exactly like those following irradiation with 257 $\mathrm{m} \mu$," should be answered affirmatively.

It would seem that the photochemical processes elicited by the two wave lengths converge to form one common stimulus to the skin capillaries. The reason for the difference in speed of appearance of 300 and $257 \mathrm{~m} \mu$ erythemata should, then, be sought in the earlier stages of the photochemical processes (Rottier, personal communication). In this connection, reference should also be made to the supposition by Blum and Terus (4), that the direct influence of $300 \mathrm{~m} \mu$ rays on the capillaries may delay the reaction to the dilatator substance.

It is probable that there is only one stimulus for melanin production, too. This stimulus may be of the same nature as that eliciting erythema, but one should bear in mind the possibility that increased melanin production may be a sequel to erythema. The latter supposition is favored by the finding of the long persistence of erythema and of the exact coincidence of the erythematous and pigmented areas.

One might well ask whether ultraviolet irradiation invariably results in increased pigmentation.
We have, in fact, come across a few instances in which, during the first few days after irradiation, a slight bleaching of the skin superimposed on the erythema is suggested by the curves. The bleaching could be localized in the epidermis, but we have not been able to find out whether in these cases the increase in reflection should be ascribed to a real diminution of pigment or to an increase of the scattering power of the epidermal layers. This phenomenon, which has also been found after local $x$-ray irradiation of the same subject, certainly merits further investigation.

\section{SUM MARY}

1. A method is described enabling small areas of the skin to be scanned with a narrow strip of monochromatic light. The skin reflection during scanning is recorded, and repeating the process at various wave lengths yields data on the spectral reflection of erythematous and pigmented fields in the area scanned and on the distribution of the colored matter. Narrow rectangular areas of erythema and of pigmentation caused by ultraviolet irradiation ( 257 or $300 \mathrm{~m} \mu$ ) are thus examined.

2. It appears that skin reactions to moderate irradiations with $257 \mathrm{~m} \mu$ and $300 \mathrm{~m} \mu$, respectively, differ only in the speed of formation of the erythema, the $257 \mathrm{~m} \mu$ erythema preceding the $300 \mathrm{~m} \mu$ erythema of comparable "strength" by several hours.

3. Ultraviolet erythema is shown to persist for at least 45 days after irradiation.

4. A very close spatial and temporal relationship has been found to exist between erythema and pigmentation after ultraviolet irradiation.

\section{ACKNOWLEDGMENTS}

The author is indebted to Prof. Berkelbach van der Sprenkel and to Dr. Rottier for constant encouragement and valuable criticism. His thanks are also due to Miss A. de Ruiter for untiring technical assistance.

\section{REFERENCES}

1. Keller, P., Uber die Wirkungen des ültravioletten Lichtes auf die Haut. Strahlentherapie, 1928, 28, 152.

2. Miescher, G., Das Problem des Lichtschutzes und der Lichtgewöhnung. Strahlentherapie, 1930, 35, 403.

3. Hamperl, H., Henschke, U., und Schulze, R., Vergleich der Hautreaktionen beim Bestrahlungserythem 
und bei der direkten Pigmentierung. Virchows f. Arch. path. Anat., 1939, 304, 19.

4. Blum, H. F., and Terus, W. S., Inhibition of the erythema of sunburn by large doses of ultraviolet radiation. Am. J. Physiol., 1946, 146, 97.

5. Rottier, P. B., Sur l'interpretation du spectre d'action de la lumière ultraviolette par l'intermédiaire de deux substances-mères photolysables, avec localisation différente dans l'épiderme. Synthèse Sémeiol. Thérap., 1951, 21, 14.

6. Rottier, P. B., and Mullink, J. A. M., Localization of erythemal processes caused by ultra-violet light in human skin. Nature, 1952, 170, 574.
7. Drabkin, D. L., and Schmidt, C. F., Spectrophotometric studies. XII. Observation of circulating blood in vivo, and the direct determination of the saturation of hemoglobin in arterial blood. J. Biol. Chem., 1945, 157, 69.

8. Edwards, E. A., and Duntley, S. Q., The pigments and color of living human skin. Am. J. Anat., 1939, $65,1$.

9. Edwards, E. A., Finkelstein, N. A., and Duntley, S. Q., Spectrophotometry of living human skin in the ultraviolet range. J. Invest. Dermat., 1951, 16, 311.

10. Schulz, A., Thermische Einflüsse auf die Erythembildung. Strahlentherapie, 1951, 86, 142.

\section{SPECIAL NOTICE TO SUBSCRIBERS}

Post Offices will no longer forward the Journal when you move.

Please notify The Journal of Clinical Investigation, Business Office, 622 West 168th Street, New York 32, N. Y. at once when you have a change of address, and do not omit the zone number if there is one. 